\title{
Advancing the Cultural Study of Personality and Identity:
}

\section{Models, Methods, and Outcomes}

\author{
Moin Syed \\ University of Minnesota \\ In press \\ Current Issues in Personality Psychology
}

Address correspondence to moin@umn.edu. Thanks to Kate McLean for helpful comments as I drafted this article. All content remains my responsibility alone. 


\begin{abstract}
Personality and identity are overlapping but distinct constructs. This article describes the three-level model of personality and, specifically, how identity fits within it. Importantly, identity operates at multiple levels of personality, depending on whether the focus is on domain-specific identity development processes or the integrated life story on a broad scale. The strength of the three level model of personality is then described with respect to a) how it provides an ideal framework for cultural psychological inquiries into universality and specificity, b) how it is well-suited for mixed methods research that combines quantitative and qualitative methods, and c) how different levels of personality may be related to psychological outcomes.
\end{abstract}

Keywords: Personality, Identity, Methods, Narrative 
Advancing the Cultural Study of Personality and Identity:

Models, Methods, and Outcomes

I am frequently asked about the distinction between personality and identity. Are they separate concepts or different terms for the same thing? Is personality part of identity, or vice versa? In some ways, it is quite surprising that these questions are seemingly still left unresolved, given that questions of personality and identity were taken up by the earliest and most influential psychological thinkers (e.g., Erikson, 1968; James, 1890).

The present special issue on personality and identity does not directly address the question on the relation between the two. As I was reading the articles, however, I was continually reminded of it, along with several other "big questions" in the field, including: How do we understand the complexity of personality and identity within a micro and macro developmental context? What aspects of personality and identity are universal vs. cultural specific? What methods should we use to study personality and identity? In what ways do personality and identity "matter" for life outcomes? None of the articles in the special issue directly address these questions either, but these are the kind of questions that arise when taking the articles as a set rather than in isolation. Thus, in this commentary I provide some thoughts on these big questions, all of which were inspired by the individual articles.

\section{Understanding The Broad Context of Personality}

There is a widespread-and unfortunate-tendency to equate personality with personality traits. This tendency is evident in several of the articles in this issue. Without a doubt, the development of the big five trait taxonomy has been an invaluable and generative structure for psychological investigations of traits (Caspi, Roberts, \& Shiner, 
2005). However, personality has always been, and always will be, a multi-level system that goes far beyond just traits (McAdams \& Olson, 2010).

To counter the increasing stranglehold of trait conceptions of personality on the field, McAdams (1995) proposed a three-level framework for understanding personality: traits, characteristic adaptations, and the integrated life story. The three levels represent three distinct aspects of personality not reducible to any other level. These three levels map on to the classic dictum in personality psychology that every person is like all other persons, like some other persons, and like no other person (Kluckhohn \& Murray, 1953). McAdams and Pals (2006) expanded this model into the "new Big Five" including evolution at level 1 and culture at level 5 . This led to the definition of personality:

Personality is conceived as (a) an individual's unique variation on the general evolutionary design for human nature, expressed as a developing pattern of (b) dispositional traits, (c) characteristic adaptations, and (d) self-defining life narratives, complexly and differentially situated (e) in culture and social context (p, 204).

Despite that expansion to five levels, the original three-level model continues to be the prime currency of personality research (Dunlop, 2015; Lilgendahl, 2015; McAdams, 2013), and is thus what I primarily focus on here. Importantly, the three-level framework is not a psychological theory, per se, in that it does not specify potentially causal relations between and among the three levels. Rather, the model is a framework that organizes different aspects of personality in conceptual space. What follows is a brief description of the three levels.

\section{Level 1 Traits}


At the most basic level of personality are traits, which are decontextualized descriptions of relatively stable individual differences. The big five trait taxonomy (neuroticism/emotional stability, extraversion, openness, agreeableness, and conscientiousness) has become widely accepted, as it has shown to be a highly generalizable model that accounts for the covariation in a large number of human individual differences (Caspi et al., 2005; DeYoung, 2015). Traits are relatively stable, but they do in fact change across the lifespan and in response to intervention (Roberts, Luo, Briley, Chow, Su, \& Hill, 2017; Roberts \& Mroczek, 2008).

Although often referred to as the big five trait taxonomy it may be more accurately labeled as the hierarchical trait taxonomy, as the big five represents just one level within a multi-level hierarchical classification system (Costa \& McRae, 1995; DeYoung, Quilty \& Peterson, 2007). Moving up one level in the hierarchy leads to the two meta-traits of stability and plasticity (DeYoung, 2010). Stability represents the tendency to express consistency, reliability, and normativity, and is defined as the shared variance of neuroticism/emotional stability, agreeableness, and conscientiousness. Plasticity, defined as the shared variance of extraversion and openness, represents a tendency towards dynamicity, novelty, and adaptiveness. These two meta-traits direct individuals' goaloriented behavior, with stability facilitating the achievement of goals within an overall functional system and plasticity facilitating the ability to adapt to unexpected occurrences in pursuit of goals (DeYoung, 2010; cf. Kunnen \& Metz, 2015; Piaget, 1954).

The third level of the hierarchy, directly below the big five traits, lies 10 aspects (DeYoung et al., 2007). With two aspects per each of the big five traits, the aspects represent potentially divergent traits within each of the big five, and thus help to unpack 
some of the coarseness of the five factor model. The fourth level of the hierarchy contains the facets (Costa \& McRae, 1995). The number of facets within each of the big five is unknown, as these represent much more specific traits. Additionally, either facets themselves can vary in specificity or there is a fifth level of the hierarchy that captures this specificity. For example, self-discipline is a facet-level trait, under the aspect of industriousness, domain of conscientious, and meta-trait of stability. But self-discipline can be taken even further to different life domains. For example, academic self-discipline which could be quite distinct from other forms of self-discipline such as financial or romantic (Jung, 2013).

As we move this far down in the trait hierarchy, however, becoming more specific and contextualized, we begin to move to level 2 of McAdams' (1995) framework. Before doing so, however, it is important to consider that, even when primarily concerned with Level 1 traits, using the big five as the default level in the hierarchy may not be optimal. It could be that specific research questions are better suited for the meta-traits or facets. Moreover, when examining trait covariation in a new cultural context, such as with the South African Personality Instrument (SAPI) used by Nel and his colleagues (this issue), it is important to include traits that fall at different levels of the hierarchy and then test a hierarchical model in order to truly assess the similarities and differences in the model.

\section{Level 2 Characteristic Adaptations}

Characteristic adaptions are the more contextualized aspects of personality, those that are situated within specific life contexts and ideological settings. Characteristic adaptions are the route through which individuals enact their traits in their daily lives, particularly as related to developmental concerns (McAdams, 2013). McAdams and Pals 
(2006) defined characteristic adaptions as variation "with respect to a wide range of motivational, social-cognitive, and developmental adaptations, contextualized in time, place, and/or social role" (p. 208). These represent developmental concerns (e.g., identity, generativity), motivations, goals, and behaviors. While of course this level of analysis is central to understanding personality, it is also somewhat nebulous and at risk for serving as a catch-all "not traits" level of personality. DeYoung (2015) made major advances in this regard by asserting that all characteristics adaptations can be considered goals, interpretations, and strategies. More specifically, he argued that, in contrast to traits, characteristics adaptations are not culturally universal, but rather constitute reactions to culturally specific demands (e.g., aggression as assessed in Kozina, this issue).

\section{Level 3 Integrated Life Story}

Level 3 of the personality system is the integrated life narrative, which consists of the stories that people tell about their personal past. Narrative psychology focuses on how individuals reason about their past experiences and how stories serve functional roles for self-understanding (McAdams, 2013). Narrating one's life allows for a sense of coherence across time and place to provide a sense of purpose and meaning. In contrast to traits and characteristic adaptations, the integrated life story emphasize an individual life lived; personality in terms of the complex and contextual ways in which traits and characteristic adaptations manifest in specific, yet potentially predictable, ways across lifetimes (Josselson \& Flum, 2015). Thus, level 3 is a highly idiographic level of personality that seeks to understand what is truly unique about a person.

\section{So Where Does Identity Fit in This System?}


The three-level framework is extremely helpful for articulating the nature and structure of personality and has been widely accepted in the field. But what about identity? Identity as a concept has not been organized into such a system, in part because it is examined by a much larger number of social science disciplines and psychological subdisciplines (Brubaker \& Cooper, 2000; Syed, Azmitia, \& Cooper, 2011; Schwartz, Vignoles, \& Luyckx, 2011). A broader question is whether it makes sense for such an organizational system to be developed, rather than folding identity into the three-level framework. Returning to McAdams and Pals' (2006) definition of personality provided earlier, it should be clear that identity is a much more specific aspect of the self, and therefore can be subsumed within the multi-level personality system. Almost any psychological definition of identity involves a conscious awareness of the self, involving some degree of reflection or personal salience about who one is, was, will be, and ought to be (e.g., Ashmore, Deaux, \& McLaughlin-Volpe, 2004; Umaña-Taylor et al., 2014; Verkuyten, 2016; Way, Santos, Niwa, \& Kim-Gervey, 2008). Additionally, any definition of identity involves change or situational variability as a central component of the construct—suggesting that identity is distinct from personality traits, which are relatively stable ${ }^{1}$. But is identity Level 2 or Level 3 ?

This question poses a major challenge to integrating identity into the personality framework, as identity is defined differently and at varying levels of analysis (Lilgendahl, 2015; McAdams, 2013). Coming from a more personality and lifespan perspective, McAdams locates identity at level 3. Individuals' identities are represented by their ability to create an integrated life story that provides meaning and purpose. This makes good

\footnotetext{
${ }^{1}$ A scholar of social identity theory may note that Tajfel's (1981) definition of personal identity, contra social identity, specifies stability. However, Tajfel defined personal identity as personality traits, which accounts for the discrepancy. This errant use of the term personal identity is important to be aware of when consuming literature on social identity, as the distinction is still used today.
} 
sense from a personality perspective, but less so from a developmental or social psychological approach to identity.

The vast majority of identity research has not focused on integrated meaning through time but rather on domain-specific and situationally bound identities (Dimitrova, Chasiotis, Bender \& Van de Vijver, this issue; Kosic \& Dimitrova, this issue; see also volumes by McLean \& Syed, 2015, and Schwartz, Vignoles, \& Luyckx, 2011). Domain-specific and situational identities are well-aligned with individual goals, interpretations, and strategies that are bound in time in context-in other words, Level 2 characteristic adaptations. Developmental research has largely made use of the identity status model, which consists of the dual processes of exploration and commitment of identities within specific domains, such as occupation, friendship, religion, ideology, ethnicity, and so on (Dimitrova et al., this issue; Crocetti \& Meeus, 2015; McLean, Syed, \& Shucard, 2016). This research has been predominantly cross-sectional, examining the structure of identity at a given point in time, or short-term longitudinal examining changes in these processes over 2-5 years (Kroger, Martinussen, \& Marcia, 2011; Meeus, 2011). Similarly, social psychological approaches to identity favor situational salience, or how certain contexts lead to the awareness of social group memberships and the subsequent behaviors that awareness engenders (Ashmore et al., 2004; Verkuyten, 2016). This work is not about integrative meaning but rather situational meaning, and how identities become relevant for immediate or near-term thoughts, feelings, and actions. All of this is clearly not Level 3.

Research on narrative identity integrates personality, development, and social aspects of identity (McAdams, 2013). Because Level 3 is the integrative life story there is a tendency to conceptualize all narrative identity research as representing level 3 (e.g., 
Dunlop, Walker, \& Wiens, 2013). But a great deal of narrative identity research is not about the integrated life story; it is about how individuals' make sense of small slices of their lives. Despite the emphasis on individuals' using narrative to integrate their past, present, and future, most narrative studies focus on links between the past and the present, with little attention to the future (McLean \& Pasupathi, 2012; Syed \& Mitchell, 2015). Indeed, this research tends to focus on specific event narratives from individuals' lives; memories of specific types of events from individuals' past (McLean \& Thorne, 2003; Singer, 2004; Syed \& Azmitia, 2010). These event narratives are then coded for structural elements (e.g., coherence), affective (e.g., positive emotion) and motivational themes (e.g., agency and communion), and autobiographical reasoning (e.g., meaning-making, self-event connections; Adler et al., 2016; Adler et al., in press). Having a single event narrative from someone's life is not really level 3 , in that it does not say much about their integrated life story. Rather, it seems like a much better fit at level 2, as the singular story reveals goals, interpretations, and strategies that are contextualized in time and context ${ }^{2}$. In contrast, Level 3 should be reserved for the more long-term, autobiographical life story construction in which individuals make meaning of their past experiences to provide a sense of personal continuity (see Josselson, 2009, for an excellent example). Thus, the key word of level 3 is not "narrative" but rather "integrated."

So, in sum, identity clearly fits within the broader three-level system of personality, but where it fits depends on the particular level that identity is being considered.

\section{Strength of the Three Levels as a Cultural Model}

${ }^{2}$ This is one reason why DeYoung's (2015) CB5T model collapses level 3 into level 2; he failed to engage with the difference between narrated life experiences and an integrated life narrative. 
Although not always explicitly recognized as such, the three-level model of personality is a cultural model of personality. The full model is entirely consistent with Shweder's (2000) conceptualization of cultural psychology as an approach that assumes psychological pluralism, in which cultures exhibit different mentalities in accordance with their contexts. Moreover, the three-level model nicely addresses the universality vs. local dilemma often invoked in cultural psychology. While Level 1 , traits, is clearly focused on universal aspects of personality, Levels 2 and 3 emphasize context-specific aspects (DeYoung, 2015; McAdams, 2013).

Defining Level 2 as goals, interpretations, and strategies indicates how they are grounded in developmental and cultural space. This is not so different from Gjerde's (2004) advocacy for focusing on individuals' concerns when conducting anti-imperial cultural psychology. To be sure, universal psychological concepts such as agency, affiliation, exploration, and so on are quintessential Level 2 constructs, and these can be deployed in research studies in a universal way (e.g., self-determination theory; Ryan \& Deci, 2000; see also Phinney \& Baldelomar, 2011, for a critique of identity research). But these concepts can—and should—be contextualized within local conditions (Jensen, 2012). For example, when designing a study on identity development of Swedish youth, Frisén and Wängqvist (2011) first conducted focus groups to determine the most appropriate identity domains in which to assess exploration and commitment. This preliminary analysis indicated that religion and dating — two domains frequently included in identity status research — were not salient to this population. Had they included these domains in their study, they would have still acquired data on Level 2 processes of identity exploration and commitment, but they would not been well-suited for the lives of their target population. What would have 
been a universalist Level 2 investigation was more appropriately developed into a cultural one. Thus, Level 2 provides an ideal context for conducting research that adheres to the cultural psychological dictum of universalism without uniformity (Shweder, 2000); or to put it another way, for blending emic and etic approaches, as discussed in He and Van de Vijver (this issue).

The focus of Level 3, the integrative life story, is on how individuals' make integrative meaning of their lives, which is about as cultural as you can get. Indeed, case studies and multiple case comparisons provide the rich, contextualized detail that cultural psychologists seek—psychological processes grounded only in individuals' own contexts (Hammack, 2006; Josseslon, 2009; McAdams, 2010; Robinson \& McAdams, 2015;

Schachter, 2004). Such an analysis can be difficult to achieve with other, more aggregative methodologies.

In sum, personality psychology is not often thought of as particularly strong as a cultural psychology. That may be true, and remain to be true, so long as personality is equated with personality traits. Opening up personality inquiries to the other two levels of analysis provides opportunities for recognizing the cultural nature of personality.

\section{Situating Methodological Diversity within the Three Levels}

As is evident from the preceding discussion of the three levels as a cultural model, situating research within this model clearly calls for methodological diversity. Beyond that banal call, the model also provides some directions for where different methods should be located, or how to best use them to understand the broad context of personality. Indeed, there is a methodological gradient that maps on to the three levels: Assessments of Level 1 traits will almost always rely on quantitative methods, Level 2 characteristic adaptations 
can be based on quantitative or qualitative methods, and Level 3 integrative life story is almost exclusively based on qualitative methods.

A major implication of this methodological gradient is that any study that truly seeks to include all three levels must rely on mixed methods. Mixed methods research has enjoyed increased acceptance within psychological research in the last few decades. Nevertheless, mixed methods designs are still relatively rare in the psychological literature, including personality psychology. But mixed methods designs are particularly well-suited for studies of personality, especially when situated within the three levels. For example, Singer (2005) has frequently combined data on traits, personal strivings (Level 2), and life stories to better understand how personality is associated with positive and negative functioning. Each level provides different, yet complementary, information to better understand the whole person-an optimal outcome for personality psychology.

Even when mixed methods studies are used for research on personality and identity, they are not often clearly situated within the methodological frameworks of mixed methods research. On selecting a good research design in cultural psychology, He and Van de Vijver (this issue) focus on quantitative concerns of bias, measurement, equivalence, validity, and so on, mentioning at the end that there is a greater need for mixed methods studies. I agree with this, but they also claim that there has not been sufficient work on different mixed methods designs and how to use them. This is most certainly not the case.

Reviewing the major mixed methods designs is beyond the scope of this article, but two types of designs are particularly worth mentioning (interested readers should consult Creswell \& Plano Clark, 2007, for an accessible introduction to mixed methods designs). Exploratory designs are ones in which a qualitative study is conducted first, followed by a 
quantitative study. This type of design is well-suited for when little is known about a topic, or when researchers are interested in developing a new measure. Indeed, Nel and colleagues (this issue) describe how the South African Personality Inventory was developed initially through conducting interviews with the diverse ethno-linguistic population, which then led to the generation of items that were subject to psychometric analyses. In contrast, explanatory designs begin with quantitative analyses and then subsequently rely on qualitative methods. This type of design is intended to provide deeper understanding about an observed quantitative finding through the qualitative analysis. For example, Tatalović Vorkapić and Peloza (this issue) found different patterns of associations between personality traits and well-being for pre-school vs. primary school teachers, but were left only to speculate as to why. Conducting follow-up interviews, focus groups, or classroom observations could help provide ideas for what might account for the differential associations. In short, mixed methods can go a long way towards developing our understanding of personality and how it is associated with life outcomes.

\section{The Three Levels and Outcomes}

A final point to consider is how the three-level model is associated with various psychological outcomes. Although there is clearly great interest in this question among researchers, there is also a great need for deeper thinking in the area across individual differences research. The selection of outcomes is often seemingly haphazard, leading Roberts (2011) to the perceptive term, outcome fragmentation grenades, to characterize how it seems as though researchers assess several different outcomes to see "what works" rather than clearly linking their psychological questions to the most theoretically sensible outcomes. Indeed, this problem interfaces with the well-documented problem of selective 
reporting of results and publication bias in favor of statistically significant results (Ferguson \& Heene, 2012). To counter this broad problem, I propose five methodological best practices with respect to studying outcomes:

Draw upon principles from personality psychology. When developing hypotheses about how personality (traits in particular) might be related to outcomes, researchers should draw upon the extensive research in this area. Indeed, based on this research a set of principles have been advanced, some of which are directly associated with outcomes (and the remainder at least indirectly so). In particular, the maturity principle, which states that individuals become more conscientious, agreeable, and emotionally stable over time (Caspi, et al., 2005), and the social investment principle, that personality develops through investment in normative social conventions and institutions (Roberts, Wood, \& Smith, 2005), both provide potentially greater predictive power than a decontextualized analysis of individual traits.

Traits are likely related to outcomes through Level 2. Because traits constitute the broadest level of personality, they are also likely to play a distal role with respect to situational psychological outcomes. That is, associations between traits and outcomes may be mediated by contextualized Level 2 goals, interpretations, and strategies that are more proximal to individuals' psychological functioning within a particular point in time.

Use broader conceptualization of outcomes. Psychological researchers, particularly identity researchers, tend to think of outcomes as separable psychological states, such as distress symptoms and well-being. However, drawing from broader philosophical conceptions of well-being illustrates how limited this view is (Tiberius, 2015). Both Levels 2 and 3 can, on their own, constitute well-being. For example, from an Eriksonian (1968) 
identity perspective having a sense of a coherence within a particular identity domain (e.g., occupational) is considered a marker of positive functioning on its own, and having an integrated identity across time and place is the essence of positive, healthy identity—both of these are taken as indicators of well-being regardless of how they correlate with measures of such.

Make better use of multivariate models. Oftentimes studies include several outcome variables, and will conduct separate ANOVAs or multiple regression analyses for each outcome. At the same, all of those outcome variables are correlated, sometimes substantially. When assessed piecemeal in this way, it is impossible to know which outcomes are actually associated with the predictors and which are just showing an association due to the unmodeled association with another outcome. This problem is easily solved by working in multivariate space, using path analysis or structural equation modeling (SEM), both of which can parse the overlap in multiple outcomes vis-à-vis the predictors. SEM is particularly valuable in that it can be used to create latent variables, such as broad internalizing and externalizing factors. In many ways, it is more straight forward to develop hypotheses at this level vs. relations to specific symptoms that are not easily separable (e.g., depression and anxiety).

Distinguish between positive well-being and psychological distress. It has been extensively argued that the absence of negative symptoms is not necessarily indicative of positive well-being. In other words, psychological distress and positive well-being should not be seen as two ends of a single continuum. This means that a) assessments of both domains should be included, as relevant, b) measures of the two should not be combined into a single index (as in Nel et al., this issue), and c) multivariate models should include 
both to develop greater specificity of relations. For example, studies of ethnic identity have indicated that it is related much more strongly with positive well-being than it is with reduction of distress symptoms (Syed \& Juang, 2014). Studies that include distress and not well-being, then, could result in misleading conclusions due to the inflated association because of the unmodeled association with well-being.

\section{Conclusion}

The present article was inspired by the collection of articles in this special issue on identity and personality. While each article stands on its own and makes a solid contribution, when they are taken together they lack the coherence that we should desire as we continue to move psychological research towards a proper cumulative science (Hedges, 1987). This is no knock on the articles in this special issue, as the same would be true of nearly any collections of articles on the same topic. I have used this opportunity to suggest that the three-level model of personality serves as the current state-of-the-art, and that future investigations should give serious thoughts to situating their personality research within this model. Doing so will serve the dual purpose of clarifying where any particular study is located within the broad universe of personality psychology, and facilitating research syntheses and efforts towards cumulativeness. 


\section{References}

Adler, J.M., Lodi-Smith, J., Philippe, F.L., Houle, I. (2016). The incremental validity of narrative identity in predicting well-being: A review of the field and recommendations for the future. Personality and Social Psychology Review, 20(2), 142-175.

Adler J. M., Dunlop, W. L., Fivush, R., Lilgendahl, J. P., Lodi-Smith, J. McAdams, D. P., McLean, K. C., Pasupathi, M., \& Syed, M. (in press). Research methods for studying narrative identity: A primer. Social Psychological and Personality Science.

Ashmore, R. D., Deaux, K., \& McLaughlin-Volpe, T. (2004). An organizing framework for collective identity: articulation and significance of multidimensionality. Psychological Bulletin, 130(1), 80.

Brubaker, R., \& Cooper, F. (2000). Beyond "identity". Theory and Society, 29(1), 1-47.

Caspi, A., Roberts, B. W., \& Shiner, R. L. (2005). Personality development: Stability and change. Annual Review of Psychology, 56, 453-484.

Costa Jr, P. T., \& McCrae, R. R. (1995). Domains and facets: Hierarchical personality assessment using the Revised NEO Personality Inventory. Journal of Personality Assessment, 64(1), 21-50.

Creswell, J. W., \& Clark, V. L. P. (2007). Designing and conducting mixed methods research.

Crocetti, E., \& Meeus, W. (2015). The identity status: Strengths of a person-centered approach. In K.C. McLean \& M. Syed (Eds.), The Oxford handbook of identity development (pp. 97-114). New York: Oxford University Press.

DeYoung, C. G. (2010). Toward a theory of the Big Five. Psychological Inquiry,21(1), 26-33. 
DeYoung, C. G. (2015). Cybernetic big five theory. Journal of Research in Personality, 56, 3358.

DeYoung, C. G., Quilty, L. C., \& Peterson, J. B. (2007). Between facets and domains: 10 aspects of the Big Five. Journal of Personality and Social Psychology, 93(5), 880.

Dunlop, W. L. (2015). Contextualized personality, beyond traits. European Journal of Personality, 29(3), 310-325.

Dunlop, W. L., Walker, L. J., \& Wiens, T. K. (2013). What Do We Know When We Know a Person Across Contexts? Examining Self-Concept Differentiation at the Three Levels of Personality. Journal of Personality, 81(4), 376-389.

Erikson, E. (1968). Identity, Youth and Crisis. New York, NY: W W Norton \& Co.

Ferguson, C. J., \& Heene, M. (2012). A vast graveyard of undead theories: Publication bias and psychological science's aversion to the null. Perspectives on Psychological Science, 7(6), 555-561.

Frisén, A., \& Wängqvist, M. (2011). Emerging adults in Sweden: Identity formation in the light of love, work, and family. Journal of Adolescent Research, 26(2), 200-221.

Gjerde, P. (2004). Culture, power and experience: Toward a person-centered cultural psychology. Human Development, 47, 138-147.

Hammack, P. L. (2006). Identity, conflict, and coexistence: Life stories of Israeli and Palestinian adolescents. Journal of Adolescent Research, 21(4), 323-369.

Hedges, L. V. (1987). How hard is hard science, how soft is soft science? The empirical cumulativeness of research. American Psychologist, 42(5), 443.

James, W. (1890). The principles of psychology. New York: Holt. 
Jensen, L. A. (2012). Bridging universal and cultural perspectives: A vision for developmental psychology in a global world. Child Development Perspectives, 6(1), 98-104.

Jossleson, R. (2009). The present of the past: Dialogues with memory over time. Journal of Personality, 77(3), 647-668.

Josselson, R., \& Flum, H. (2015). Identity status: On refinding the people. In K.C. McLean \& M. Syed (Eds.), The Oxford handbook of identity development. New York: Oxford University Press.

Jung, K. R. (2013). The mediational effect of academic self-discipline (ASD) between academic self-efficacy (ASE) and college GPA. Unpublished doctoral dissertation, University of Minnesota.

Murray, H. A., \& Kluckhohn, C. (1953). Personality in nature, society, and culture. New York: AA Knopf.

Kroger, J. Martinussen, M., \& Marcia, J. E. (2011). Identity stat change during adolescence and young adulthood: A meta-analysis. Journal of Adolescence, 12, 683-98.

Kunnen, E. S., \& Metz, M. (2015). Commitment and exploration: The need for a developmental approach. In K. C. McLean \& M. Syed (Eds.), The Oxford handbook of identity development (pp. 115-131). New York: Oxford University Press.

Lilgendahl, J. P. (2015). The Dynamic Role of Identity Processes in Personality Development. In K.C. McLean \& M. Syed (Eds.), The Oxford handbook of identity development. New York: Oxford University Press.

McAdams, D. P. (1995). What do we know when we know a person? Journal of Personality, 63(3), 365-396. 
McAdams, D. P. (2011). George W. Bush and the redemptive dream: A psychological portrait. New York: Oxford University Press.

McAdams, D. P. (2013). The psychological self as actor, agent, and author. Perspectives on Psychological Science, 8(3), 272-295.

McAdams, D. P., \& Olson, B. D. (2010). Personality development: Continuity and change over the life course. Annual Review of Psychology, 61, 517-542.

McAdams, D. P., \& Pals, J. L. (2006). A new Big Five: Fundamental principles for an integrative science of personality. American Psychologist, 61, 204-217.

McLean, K. C., \& Pasupathi, M. (2012). Processes of identity development: Where I am and how I got there. Identity, 12(1), 8-28.

McLean, K.C., Syed, M., \& Shucard, H. (2016). Bringing identity content to the fore: Links to identity development processes. Emerging Adulthood, 4(5), 356-364.

McLean, K. C., \& Thorne, A. (2003). Adolescents' self-defining memories about relationships. Developmental Psychology, 39, 635-645.

Meeus, W. (2011). The study of adolescent identity formation 2000-2010: A review of longitudinal research. Journal of Research on Adolescence, 21, 75-94.

Phinney, J. S., \& Baldelomar, O. A. (2011). Identity development in multiple cultural contexts. In L. A. Jensen (Ed.)., Bridging cultural and developmental approaches to psychology: New syntheses in theory, research, and policy (161-186).

Piaget, J. (1954). The construction of reality in the child. New York: Basic Books.

Roberts (2011, November). Personality psychology has a serious problem (and so do many other areas of psychology). P: The Online Newsletter for Personality Science, 6. 
Roberts, B. W., Luo, J., Briley, D. A., Chow, P. I., Su, R., \& Hill, P. L. (2017). A systematic review of personality trait change through intervention. Psychological Bulletin, 143(2), 117141.

Roberts, B. W., \& Mroczek, D. (2008). Personality trait change in adulthood. Current Directions in Psychological Science, 17(1), 31-35.

Roberts, B. W., Wood, D., \& Smith, J. L. (2005). Evaluating five factor theory and social investment perspectives on personality trait development. Journal of Research in Personality, 39(1), 166-184.

Robinson, O. C., \& McAdams, D. P. (2015). Four functional roles for case studies in emerging adulthood research. Emerging Adulthood, 3(6), 413-420.

Ryan, R. M., \& Deci, E. L. (2000). Self-determination theory and the facilitation of intrinsic motivation, social development, and well-being. American Psychologist, 55(1), 68.

Schachter, E. P. (2004). Identity configurations: A new perspective on identity formation in contemporary society. Journal of Personality 72 (1), 167-200.

Schwartz, S. J., Luyckx, K., \& Vignoles, V. L. (Eds.) (2011). Handbook of identity theory and research. New York: Springer.

Shweder, R. A. (2000). The psychology of practice and the practice of the three psychologies. Asian Journal of Social Psychology, 3(3), 207-222.

Singer, J. A. (2004). Narrative identity and meaning-making across the lifespan: An introduction. Journal of Personality, 72, 437-460.

Singer, J. A. (2005). Personality and psychotherapy: Treating the whole person. Guilford Press. 
Syed, M., \& Azmitia, M. (2010). Narrative and ethnic identity exploration: A longitudinal account of emerging adults' ethnicity-related experiences. Developmental Psychology, 46(1), 208-219.

Syed, M., Azmitia, M., \& Cooper, C. R. (2011). Identity and academic success among underrepresented ethnic minorities: An interdisciplinary review and integration. Journal of Social Issues, 67(3), 442-468.

Syed, M., \& Juang, L. P. (2014). Ethnic identity, identity coherence, and psychological functioning: Testing basic assumptions of the developmental model. Cultural Diversity and Ethnic Minority Psychology, 20(2), 176-190.

Syed, M., \& Mitchell, L. L. (2015). Temporal identity integration as a core developmental process. In R. Scott, \& S. Kosslyn (Eds.). Emerging Trends in the Social and Behavioral Sciences (pp. 1-15). San Francisco, CA: Wiley.

Tajfel, H. (1981). Human groups and social categories: Studies in social psychology.

Tiberius, V. (2015). Prudential value. In I. Hirose \& J. Olson (Eds.), The Oxford handbook of value theory. New York: Oxford University Press.

Umaña-Taylor, A. J., Quintana, S. M., Lee, R. M., Cross, W. E., Rivas-Drake, D., Schwartz, S. J., Syed, M., Yip, T., Seaton, E., \& Ethnic/Racial Identity Study Group. (2014). Ethnic and racial identity revisited: An integrated conceptualization. Child Development, 85(1), 21-39.

Verkuyten, M. (2016). Further Conceptualizing Ethnic and Racial Identity Research: The Social Identity Approach and Its Dynamic Model. Child Development, 87(6), 17961812. 
Way, N., Santos, C., Niwa, E. Y., \& Kim-Gervey, C. (2008). To be or not to be: An exploration of ethnic identity development in context. New Directions for Child and Adolescent Development, 2008(120), 61-79. 